\title{
A young man with oligoarthritis preceded by urethritis and diarrhea
}

\author{
Ahmed Zedan MD, Imran Umer MD, Yasir Ahmed MD, Donald Loveman MD
}

\begin{abstract}
Reactive arthritis is a form of seronegative spondyloarthritis temporally triggered by an apparent infection, usually gastroenteritis or urethritis. This arthritis typically starts within a few weeks of the inciting infection. It commonly affects young adults and is strongly associated with the MHC class I antigen HLA-B27. Here we present an interesting case of young man with HLA-B27 positive reactive arthritis in whom the presumptive diagnosis was reached early in the course of his illness.
\end{abstract}

Key words: Reactive arthritis, oligoarthritis, chlamydial urinary tract infection, HLA-B27 positive arthritis

\section{INTRODUCTION}

Reactive arthritis is an autoimmune disease which usually develops soon after or during a presumptive gastrointestinal or urogenital infection. ${ }^{1}$ Typically reactive arthritis presents with asymmetric oligoarthritis that mainly involves lower limbs. ${ }^{2}$ Here we present an interesting case of young man who presented with reactive arthritis after gastro-urogenital symptoms.

\section{Case Presenttion}

A 20-year-old man with Gilbert's syndrome was admitted with left groin pain for one week. He had an episode of loose watery diarrhea associated with abdominal pain two weeks previously. His diarrhea resolved in three days. Subsequently he noticed dysuria with blood in the urine but no fever or chills.

Corresponding author: Yasir Ahmed MD

Contact Information: Yasir.ahmed@ttuhsc.edu

DOI: 10.12746/swrccc2014.0208.106
He was treated with oral ciprofloxacin. Subsequently he was switched to nitrofurantoin and cephalexin without any improvement. Two days thereafter he noted redness in both eyes and the new onset of pain in the left groin. By the time of his arrival to our facility his ocular redness had resolved. His roommate had also experienced a self-limited diarrheal illness.

Physical examination revealed temperature $98^{\circ} \mathrm{F}$, heart rate 110 beats/minute, respiratory rate 16 breaths/minute, and blood pressure 110/67 mmHg. Examination of his left hip joint demonstrated tenderness over the groin and a restricted range of motion. The remainder of the musculoskeletal and systemic examination was unremarkable with the exception of a small amount of purulent discharge from the urethra.

The patient underwent a left hip joint arthrocentesis on the day of admission. On hospital day three his left hip pain was slightly improved, but he developed pain and swelling in the left ankle joint. On 
hospital day four pain and swelling of left wrist joint developed. Laboratory work revealed hemoglobin $14.2 \mathrm{gm} / \mathrm{dl}$, white blood cell count $8,200 / \mathrm{mm}^{3}$, platelet count $325,000 / \mathrm{mm}^{3}$, and C-reactive protein (CRP) 4.7 $\mathrm{mg} / \mathrm{dl}$ (normal : $<1.0 \mathrm{mg} / \mathrm{dl}$ ); electrolytes, liver, and renal function tests were normal. Left hip joint synovial fluid showed white blood cell count of $18,500 / \mathrm{mm}^{3}$ with $79 \%$ neutrophils. Tests for infection, including hepatitis $A, B$ and $C$ screening tests, monospot test, rapid group $A$ streptococcus pharyngeal smear, human immune deficiency virus (HIV) screening, blood and synovial fluid Gram stain, bacterial culture, fungal culture, urine culture, polymerase chain reaction (PCR) for Chlamydia trachomatis and Neisseria gonorrhoeae, and Campylobacter jujeni antibody IgG, were negative. Stool specimens for Salmonella spp, Shigella spp, Campylobacter spp, Clostridium difficile PCR, and Giardia lamblia antigen were also negative. Immunologic work up, including rheumatoid factor, anti-cyclic citrullinated peptide immunoglobulin $\mathrm{G}(\lg G)$, double stranded deoxyribonucleic acid (dsDNA), anti-nuclear antibodies (ANA) IgG by ELISA, and DNase $B$ antibody, were normal. HLA-B27 antigen was positive. Magnetic resonance imaging showed arthritis of the left wrist joint and some evidence of left Achilles tendinitis.

The patient was initially started on empiric intravenous vancomycin and ceftriaxone until his blood cultures, left hip synovial fluid Gram stain, and urethral cultures were reported negative. Oral prednisone $20 \mathrm{mg}$ daily was added along with naproxen $500 \mathrm{mg}$ twice a day. His joint symptoms improved. He was discharged on a tapering dose of prednisone, naproxen, and a short course of empiric oral doxycycline plus rifampin. At an outpatient visit four weeks after discharge he had again developed pain in the left wrist and ankle. Low dose prednisone along with weekly methotrexate $15 \mathrm{mg}$ and daily folic acid $1 \mathrm{mg}$ was started. His symptoms are well controlled till now at nine weeks post diagnosis.

\section{Discussion}

Reactive arthritis is a form of seronegative spondyloarthritis triggered by infection, usually gastroenteritis or urethritis. However, blood and synovial culture workup often remains negative. ${ }^{1}$ Nevertheless certain microorganisms, most commonly Chlamydia trachomatis, Yersinia spp, Salmonella spp, Shigella spp, Campylobacter spp, and Escherichia coli, have been strongly associated with reactive arthritis. ${ }^{2}$ Reactive arthritis commonly affects persons between 15 to 35 years of age.

The clinical presentation of reactive arthritis typically occurs one to four weeks after an inciting infection. ${ }^{4}$ The patient usually presents with asymmetrical oligoarthritis involving the lower limbs, but the upper limbs joints can be involved as seen in our patient. Arthritis can also be associated with enthesitis, inflammation at the insertion site of ligaments or tendons. Extra-articular manifestations include conjunctivitis, erythema nodosum, keratoderma blenorrhagica, circinate balanitis, and mucosal ulcers. Overall $10 \%$ of patients with reactive arthritis develop cardiac manifestations, and these are more common in patients with chronic disease. ${ }^{6}$ Pericarditis, aortic valve insufficiency, conduction block, and rarely cardiogenic shock have been reported cardiac manifestations in the literature. ${ }^{7,89}$ The average duration of acute reactive arthritis is three to five months. Arthritis for more than six months is considered a sign of chronicity.

The diagnosis of reactive arthritis is made by the presence of asymmetric oligoarthritis preceded by symptoms of gastrointestinal or urogenital infection in the absence of other causes of arthritis. There are no definite diagnostic laboratory tests or radiographic findings in reactive arthritis. Erythrocyte sedimentation rate (ESR) is markedly elevated in most cases, and values above $60 \mathrm{~mm} / 1$ st hour are commonly seen. Complement C3 and C4 levels and CRP are elevated, especially at the onset of the disease. ${ }^{3}$ There may be mild leukocytosis and anemia in the early phase of the disease. HLA-B 27 antigen is positive in most cases, and rheumatoid factor is consistently 
negative.

In general, antibiotics are not indicated for uncomplicated enteric infections or for treatment of the reactive arthritis itself. Antibiotic therapy should be used for treatment of active urinary tract infection with Chlamydia trachomatis. ${ }^{12}$ Non-steroidal anti-inflammatory drugs (NSAIDs) are the first line and the cornerstone treatment, especially if used in full dose in the early phases of reactive arthritis. ${ }^{2,11}$ Systemic or intraarticular glucocorticoids are generally used if the patient is not responding well to NSAIDS. Disease-modifying anti-rheumatic drugs (DMARDS) are reserved for patients who are not responding to NSAIDs and require high doses of corticosteroids. Sulfasalazine and methotrexate are the most commonly used medications in this group. TNF inhibitors are used for patients resistant to corticosteroids and DMARDS. ${ }^{13}$

The prognosis of reactive arthritis depends on causative organism, the presence or absence of HLAB27, gender, and the presence of recurrent arthritis. ${ }^{14}$ HLA-B27 antigen has been associated with reactive arthritis in about $70-80 \%$ patients and predicts more severe disease. ${ }^{2,11}$ Leirisalo-Repo et al reported a retrospective study of 63 patients with a mean follow-up of 11 years. HLA-B 27 antigen was positive in $88 \%$ of the patients. He found that $16 \%$ of the patients developed a chronic course and they were all HLA-B 27 positive. ${ }^{15}$ Our patient presented with classic symptoms of reactive arthritis with positive HLA-B27 antigen and elevated CRP. All other laboratory investigations were normal. He failed to respond to NSAIDs and steroids, so methotrexate was added. The patent's symptoms are now controlled, and he continues follow with a rheumatologist.

In summary, reactive arthritis is a common autoimmune disease that should be suspected in young patients presenting with asymmetric oligoarthritis preceded by gastrointestinal or urogenital infection. The diagnosis of reactive arthritis is made by the exclusion of other known causes of arthritis, especially septic arthritis and supported by the presence of extra-articular manifestations, including conjunctivitis, urethritis, and tendonitis as seen in our case.
Author Affiliation: Ahmed Zedan and Imran Umer are residents in Internal Medicine at Texas Tech University Health Science Center in Odessa, TX. Yasir Ahmed is a faculty member in Infectious Diseases at TTUHSC in Odessa, TX. Donald Loveman is a faculty member in rheumatology at TTUHSC in Odessa, TX.

Received: 09/07/2014

Accepted: 09/23/2014

Reviewers: Vaqar Ahmed MD

Published electronically: 10/15/2014

Conflict of Interest Disclosures: none

\section{REFERENCES}

1. Ahvonen P, Sievers K, Aho K. Arthritis association with Yersinia enterocolitis infection. Acta Rheumatol Scand 1969:15:232. 2. Colmegna I, Cuchacovich R, Espinoza LR. HLA-B27-associated reactive arthritis: pathogenetic and clinical considerations. Clin Microbiol Rev 2004; 17:348-369.

3. Chun P, Kim YJ, Han YM, Kim YM. A case of reactive arthritis after Salmonella enteritis in in a 12-year-old boy. Korean $J$ Pediatr 2011 Jul; 54(7):313-5. doi: 10.3345/kjp.2011.54.7.313. Epub 2011 Jul 31.

4. Rohekar S, Pope J. Epidemiologic approach to infection and immunity: the case of reactive arthritis. Curr Opin Rheumayol 2009: 21:386.

5. Hannu T. Reactive arhrtitis. Best Pract Res Clin Rheumatol 2011 Jun; 25(3):347-57. doi: 10.1016/j.berh.2011.01.018.

6. Selmi C, Gershwin ME. Diagnosis and classification of reactive arthritis. Autoimmun Rev 2014 Apr-May; 13(4-5):546-9. doi: 10.1016/j.autrev.2014.01.005. Epub 2014 Jan 10.

7. Deer T, Rosencrance JG, Chillag SA. Cardiac conduction manifestations of Reiter's syndrome. South Med J 1991 Jun; 84(6):799-800.

8. Csonka GW, Oates JK. Pericarditis and electrocardiographic changes in Reiter's syndrome. Br Med J 1957 Apr 13; 1(5023):866-9.

9. Brown LE, Forfia P, Flynn JA. Aortic insufficiency in a patient with reactive arthritis: case report and review of the literature. HSS J 2011 Jul; 7(2):187-9. doi: 10.1007/s11420-010-9184-x. Epub 2011 Jan 14.

10. Leirisalo M, Skylv G, Kousa M, Voipio-Pulkki LM, Suoranta $\mathrm{H}$, Nissila M, et al. Followup study on patients with Reiter's disease and reactive arthritis, with special reference to HLA-B27. Arthritis Rheum 1982; 25:249-59. 
11. Leirisalo-Repo M. Reactive arthritis. Scand $J$ Rheumatol 2005 Jul-Aug; 34(4):251-9.

12. Kvien TK, Gaston JS, Bardin T, et al. Three month treatment of reactive arthritis with azithromycin: a EULAR double blind, placebo controlled study. Ann Rheum Dis 2004; 63:1113

13. Braun, J., Z. Yin, I. Spiller, S. Siegert, M. Rudwaleit, L. Liu, A. Radbruch, and J. Sieper. 1999. Low secretion of tumor necrosis alpha but no other Th1 or Th2 cytokines by peripheral blood mononuclear cells correlates with chronicity in reactive arthritis. Arthritis Rheum 42:2039-2044.

14. Schumacher, H. R., Jr. Reactive arthritis. Rheum Dis Clin North Am 1998; 24:261-273

15. Leirisalo-Repo M, Helenius $P$, Hannu T, Lehtinen A, Kreula $\mathrm{J}$, Taavitsainen $\mathrm{M}$ et al. Long-term prognosis of reactive salmonella arthritis. Annals Rheum Dis 1997; 56:516-20. 